\title{
A MICROSTRIP POWER INVERSION ARRAY USING PARASITIC ELEMENTS
}

\author{
Robert J. Dinger \\ Michelson Laboratory, Physics Division \\ Naval Weapons Center, China Lake, California 93555
}

\section{Introduction}

This paper reports recent results of experimental measurements and theoretical analysis of a reactively steered adaptive array (RESAA) comprised of microstrip patch antenna elements. A RESAA achieves pattern control by adjusting the terminating reactances on parasitic elements that are closely coupled to a single driven element; only the driven element is physically connected to a receiver by a transmission line. The value of each terminating reactance determines the phase of the signal reflected (in free space) from that parasitic element to the single receiving element. Thus, the driven antenna element "sums" the various reflected signals to form the array output. Some preliminary measurements on this type of adaptive antenna array were presented at the 1982 AP-S/URSI Symposium [1]; a more complete discussion of the results presented here can be found in [2].

\section{Experimental Results}

A five-element Iinear array was fabricated with rectangular microstrip patch elements resonant at $4.0 \mathrm{GHz}$. The edge separation was $0.1 \lambda$, and the center-to-center separation was $0.23 \lambda$. $\left(\lambda_{0}=\right.$ free space wavelength). The variable reactive load on the parasitic elements was provided by a microstrip reflection phase shifter of standard design that used a single varactor diode. Figure 1 is a photograph of the array, showing the four phase shifters connected to the feeds of the four parasitic elements.

Figure 2 displays a sample antenna pattern for this array taken in an anechoic chamber. To obtain this pattern, the array was oriented to place the test signal at the angle of incidence indicated by the arrow, and the received signal power was minimized by (manual) iterative adjustment of the phase shifter control voltages. When no further reduction was possible, the control voltages were frozen, and the pattern was measured using the test signal. In this power inversion mode of operation, the array is able to steer a null with a depth of $30 \mathrm{~dB}$ and a width of 25 degrees towards an interference signal over nearly the full aperture of the array. The reactive load values for a null at one angle are not unique, so that the features of the pattern at angles removed from the null can be controlled by placing other constraints on the choice of reactive loads. Measurements are currently underway to investigate pattern formation when a desired signal is also present. Also in progress is the implementation, using a minicomputer, of the algorithm described below.

U.S. Government work not protected by U.S. copyright. 


\section{Array Theory}

The theory developed in [3] for a reactively steered aperture array can be used to derive the following equation for the array pattern:

$$
\begin{array}{r}
F(\theta)=\frac{2 \sin \left(k_{0} h \cos \theta / 2\right) \cos \left(k_{o} L \cos \theta / 2\right)}{k_{0} h \cos \theta} \\
\times \quad \sum_{n=I}^{N}\left\{\left[z_{A}+z_{L}\right]^{-1}\right\}_{n p} e^{j k_{0} x_{n} \cos \theta}
\end{array}
$$

where $\theta$ is the pattern angle measured from broadside, $h$ is the substrate thickness, $I$ is the antenna width, and $x_{n}$ is the $x$-coordinate of the $n^{\text {th }}$ element. The matrix $\left[z_{A}\right]$ is the mutual impedance matrix of the array, and $\left[z_{L}\right]$ is a diagonal matrix of the reactive loads. The load matrix has the entries $\left(z_{L}\right)_{i j}$ $j \mathrm{X}_{\mathrm{Li}} \delta_{1 j}$, except that $\left(\mathrm{Z}_{\mathrm{L}}\right)_{\mathrm{pp}}=0$, corresponding to the driven element. The notation \{\}$_{n p}$ denotes the $n{ }^{\text {th }}$ element of the
matrix in the brackets. Figure 3 is a plot of $F(\theta)$ as a function of the reactive loads on the two parasitic elements for a three-element array. The configuration (patch size, separation, etc.) is the same as the experimental array, and the values of $\left(\mathrm{Z}_{A}\right)$ are derived from experimental measurements. The surface in Figure 3 can be viewed as an error surface; the manual iteration of the reactive loads in the experimental measurements searches this surface and finds the botton of the bowl, which corresponds to a minimun output.

\section{Adaptive Control Simulation}

We have simulated several adaptive control techniques, including a steepest descent algorithm and a random search algorithr. For discrete steepest descent control, the reactive load at step $(j+1)$ is derived from the load at step $j$ by the equation

$$
\mathrm{x}_{\mathbf{i}}(j+1)=\mathrm{x}(\mathrm{j})-\mathrm{k} \nabla_{\mathrm{x}_{i}} \varepsilon
$$

where $\mathrm{K}$ is a constant that controls the convergence rate, and $\varepsilon$ is the (N-1)-dimensional error surface (of which Figure 3 is an example). The quantity $\varepsilon$ can be estimated both in simulations and in a practical array by imposing a dither signal on the reactive load and detecting the change in the array output.

We have used Eqs. (1) and (2) to simulate the performance of a microstrip RESAA on a digital computer. As an example of the simulation results, in Figure 4 we plot the decrease in interference power and the change in the reactive loads during 
steepest descent processing for the configuration used in Figure 3. Curves are shown for two different values of $K$. In general, the simulations reproduce the behavior of the RESAA observed during the manual adaptation measurenents.

\section{Conclusions}

The microstrip reactively steered adaptive array demonstrates that a useful degree of pattern control can be achieved by adjustment of the reactive loads on parasitic elements. The main advantage of a RESAA, as compared with a conventional adaptive array, is the elimination of the mixers and other hardware needed to perform the complex weighting of each element's output at an IF. Another advantage is the necessarily smaller overall size needed to maintain tight coupling between the elements.

\section{References}

1. R. J. Dinger, "Adaptive Microstrip Antenna Array Using Reactively Terminated Parasitic Elements," 1982 AP-S/URSI Symposium.

2. R. J. Dinger, "Reactively Steered Adaptive Array (RESAA) Using Microstrip Patch Elements at $4.0 \mathrm{GHz}$," Naval Weapons Center Technical Publication (in press).

3. J. Luzwick and R. F. Harrington, "A Reactively Loaded Aperture Antenna Array," IEEE Trans. Antennas and Propagation, Vol. AP-26, PP. 543-547, July 1978.

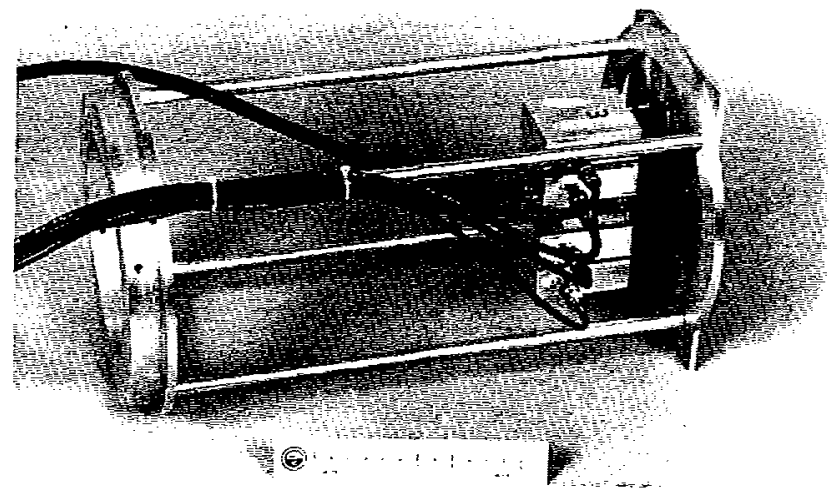

Figure 1. Rear View of Array 


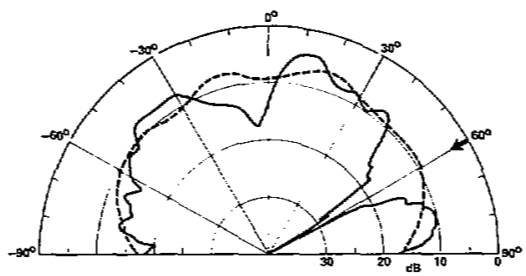
FREQUEATCY - $1.0 \mathrm{GH}$ GH
6 ELEMENT ARRAY

Figure 2. Measured Antenna Patterns

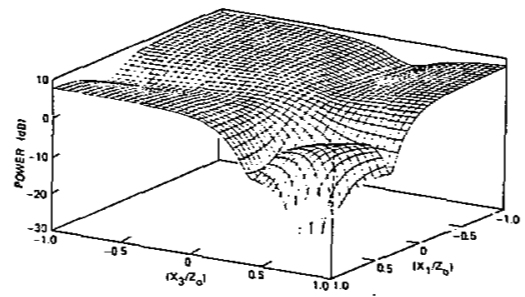

Figure 3. Plot of Equation (1)

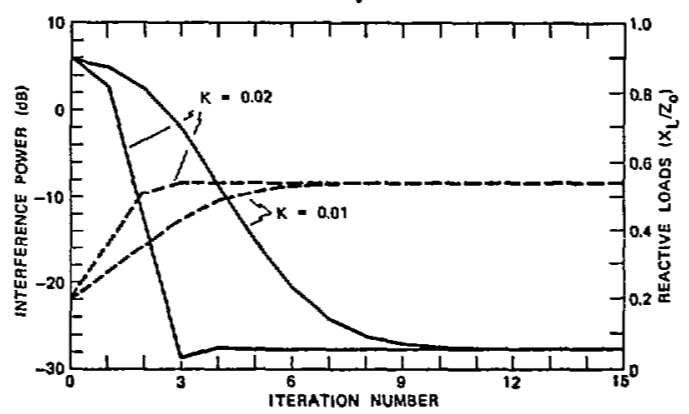

Figure 4. Simulation Results. The dashed curve is the change is reactive load (righe hand scale), and the solid curve is the change in interference power (left hand scale). 